\title{
A study of atmospheric gravity waves and travelling ionospheric disturbances at equatorial latitudes
}

\author{
R. L. Balthazor, R. J. Moffett \\ Upper Atmosphere Modelling Group, School of Mathematics and Statistics, University of Sheffield, Sheffield S3 7RH, UK
}

Received: 15 November 1996 / Revised: 4 March 1997 / Accepted: 11 March 1997

\begin{abstract}
A global coupled thermosphere-ionosphereplasmasphere model is used to simulate a family of large-scale imperfectly ducted atmospheric gravity waves (AGWs) and associated travelling ionospheric disturbances (TIDs) originating at conjugate magnetic latitudes in the north and south auroral zones and subsequently propagating meridionally to equatorial latitudes. A 'fast' dominant mode and two slower modes are identified. We find that, at the magnetic equator, all the clearly identified modes of AGW interfere constructively and pass through to the opposite hemisphere with unchanged velocity. At F-region altitudes the 'fast' AGW has the largest amplitude, and when northward propagating and southward propagating modes interfere at the equator, the TID (as parameterised by the fractional change in the electron density at the F2 peak) increases in magnitude at the equator. The amplitude of the TID at the magnetic equator is increased compared to mid-latitudes in both upper and lower F-regions with a larger increase in the upper F-region. The ionospheric disturbance at the equator persists in the upper F-region for about 1 hour and in the lower F-region for 2.5 hours after the AGWs first interfere, and it is suggested that this is due to enhancements of the TID by slower AGW modes arriving later at the magnetic equator. The complex effects of the interplays of the TIDs generated in the equatorial plasmasphere are analysed by examining neutral and ion winds predicted by the model, and are demonstrated to be consequences of the forcing of the plasmasphere along the magnetic field lines by the neutral air pressure wave.
\end{abstract}

\section{Introduction}

The relationship between atmospheric gravity waves (AGWs) and travelling ionospheric disturbances (TIDs)

Correspondence to: R. L. Balthazor has been documented in detail, with notable papers by Hines (1960), Hines (1974), Richmond (1978), Hunsucker (1982) and Jing and Hunsucker (1993), and reviewed most recently by Hocke and Schlegel (1996). The TID is the signature in the ionosphere of the passage of the AGW, the ions being forced along the field lines by the neutral air winds driven by the pressure wave. Various techniques have evolved for studying TIDs such as ionosonde and radar (e.g. Williams et al., 1988; Fukao et al., 1993), satellite (e.g. Johnson et al., 1995) and tomographic techniques (e.g. Pryse et al., 1995), and have cast light on the validity of theoretical and numerical models (e.g. Millward et al., 1993b; Kirchengast et al., 1996).

However, there has been a relative scarcity of investigations of AGWs and TIDs at equatorial latitudes. Ionospheric irregularities at equatorial latitudes have been examined recently by Hajkowicz (1995) and Bowman (1995). These effects have been previously explained as AGW 'seeding' of equatorial spread-F (Kelley, 1989). Hocke and Schlegel (1996) review the results of several observational campaigns, including those observing near, at or across the equator (Thome, 1968; Sterling et al., 1971; Hajkowicz and Hunsucker 1987) and Hajkowicz and Hunsucker (1987) suggest that, in the case of AGWs and associated TIDs approaching the equator from both hemispheres, "it is likely that both TIDs produce a constructive interference effect at the points of their encounter near the equator". Large-scale ionospheric disturbances propagating equatorwards and associated with low-latitude aurorae have been studied by Igarashi et al. (1991). Satellite measurements (Gross et al., 1984) reveal the presence of standing waves in the equatorial ionosphere, "possibly as a result of interference between waves from two sources (both auroral regions)" and Hajkowicz (1991) has shown that geomagnetic activity can produce conjugate AGWs travelling from both auroral zones. Fesen et al., (1989) used separate thermospheric and ionospheric models to examine the effects of a geomagnetic storm and showed that the equatorial disturbances observed were the result of neutral winds and AGWs. 
This study simulates simultaneous ion-velocity bursts in both north and south auroral zones at conjugate magnetic latitudes. The propagation of the resultant AGWs is examined, with emphasis on the TIDs produced at equatorial latitudes. The authors note here that whilst TIDs do not technically 'propagate' (as they are the signatures in the ionosphere of the propagating gravity wave), phrases such as 'the propagation of the TID' have become embedded in the literature. Whilst we have taken pains to avoid this inaccuracy, it is perhaps inevitable that such phrases creep into usage. We emphasise, however, that it is the AGWs that propagate, and the TIDs are merely a signature.

\section{Model description}

The Sheffield/UCL/SEL global model of the coupled thermosphere - ionosphere - plasmasphere has previously been described by Millward et al. (1993b) and more extensively by Fuller-Rowell et al. (1996) and Millward et al. (1996). In brief, coupled equations of momentum, energy and continuity are solved at fixed grid points to calculate values of density, temperature, and velocity of the neutral atmosphere, and of the $\mathrm{O}^{+}$ and $\mathrm{H}^{+}$ions in open flux tubes at high latitudes and closed flux tubes in the plasmasphere. The closed flux tubes are aligned along an eccentric dipole approximation to the Earth's magnetic field, arranged such that each flux tube returns to its starting position in a 24 hour period. Concentrations of $\mathrm{N}^{+}, \mathrm{O}_{2}^{+}, \mathrm{NO}^{+}$and $\mathrm{N}_{2}^{+}$are derived from chemical equilibrium considerations. The model output resolution is $2^{\circ}$ in latitude and $18^{\circ}$ in longitude, co-rotating with the Earth and defining a spherical polar coordinate system. Vertically, atmospheric parameters are output at fifteen fixed pressure levels, each at a separation of one scale height and the lowest at a boundary defined to be $1 \mathrm{~Pa}$ at $80 \mathrm{~km}$. The fully-coupled thermosphere - ionosphere - plasmasphere model takes into account non-uniform temperature and wind, the viscous nature of the atmosphere, and Coriolis effects.

The model was run until steady-state equilibrium (in a diurnal sense) was obtained, for January 1 conditions. A Foster electric field model (Foster, 1983) was used with an $\mathrm{F}_{10.7}$ index of 165 and TIROS precipitation activity level $7\left(\mathrm{~K}_{p} \sim 3\right)$ (Fuller-Rowell and Evans, 1987), and these were fixed during the simulations. This steady-state atmosphere was used as input conditions for the study, commencing at 12 UT.

A disturbance in the atmosphere was created by introducing a geographically co-rotating enhanced zonal electric field over a localised area in the midnight sector to simulate a simultaneous enhancement of both auroral electrojets. The extent of the enhanced electric field was $72^{\circ}$ zonally $(\sim 2500 \mathrm{~km})$ and $10^{\circ}$ meridionally $(\sim 1100 \mathrm{~km})$ with a ramped distribution in both latitude and longitude; Table 1 shows the magnitude of the enhancement to the electric field. Both northern and southern enhancements were centred on the geographic line of longitude $162^{\circ} \mathrm{E}$ to lie in the midnight sector; to
Table 1. Electrin field enhancements (in $\mathrm{mV} / \mathrm{m}$ ) at grid points around geographic centre $C_{\text {lat }}, C_{\text {lon }}$

\begin{tabular}{lclrll}
\hline & $C_{\text {lon }}-36^{\circ}$ & $C_{\text {lon }}-18^{\circ}$ & $C_{\text {lon }}$ & $C_{\text {lon }}+18^{\circ}$ & $C_{\text {lon }}+36^{\circ}$ \\
\hline$C_{\text {lat }}+4^{\circ}$ & 6 & 12 & 20 & 12 & 6 \\
$C_{\text {lat }}+2^{\circ}$ & 21 & 42 & 70 & 42 & 21 \\
$C_{\text {lat }}^{\circ}$ & 30 & 60 & 100 & 60 & 30 \\
$C_{\text {lat }}-2^{\circ}$ & 30 & 60 & 100 & 60 & 30 \\
$C_{\text {lat }}-4^{\circ}$ & 21 & 42 & 70 & 42 & 21 \\
$C_{\text {lat }}-6^{\circ}$ & 6 & 12 & 20 & 12 & 6 \\
\hline
\end{tabular}

coincide with the auroral zones, the northern enhancement was centred at $70^{\circ} \mathrm{N}$ geographic and the southern at $54^{\circ} \mathrm{S}$ geographic. It is noted that although the field lines at $162^{\circ} \mathrm{E}$ are not orientated N-S, the actual displacement from being geographically meridional is smaller than the relatively coarse $18^{\circ}$ zonal resolution of the thermospheric and ionospheric models. The instantaneous $\mathrm{E} \times \mathrm{B}$ ion drift velocity corresponding to an electric field enhancement of $100 \mathrm{mV} / \mathrm{m}$ is about $2 \mathrm{~km} \mathrm{~s}^{-1}$ in the F-region. The duration of the electric field enhancement was 10 min from 12.15 UT (23.03 LT). The magnitude, duration and extent of the enhanced electric field was designed to simulate a typical 'burst' in the auroral ion velocity (Hajkowicz 1990; Millward et al., 1993a). The geographic location of the enhancement was chosen to ensure that the AGW propagated through the night ionosphere where the amplitudes of observed TIDs are largest (Hajkowicz, 1990).

Although studies in high- and mid-latitudes have shown (Lewis et al., 1996) that there is likely to be a periodicity in the source mechanism of AGWs in the auroral electrojet, the present simulation has only considered a single 'burst' of enhancement of the electric field. This ensures that the far field effects will be limited to at most one or two cycles such that the effects of the AGW on the plasmasphere can be more clearly identified.

\section{Results and Discussion}

\subsection{The signature of the $A G W$ in the thermosphere}

An AGW is essentially a pressure wave travelling through the thermosphere and thus may be characterised directly by the effect on a fixed pressure surface in the atmosphere. Observed characteristics more commonly used as AGW parameters (such as temperatures and winds) are secondary effects of the pressure wave, whereas the change in height of a fixed pressure level is a more 'direct' measure of the wave. We have therefore chosen to parameterise the AGW by examining the (fractional) changes in height of fixed pressure surfaces in the atmosphere (Fuller-Rowell, 1984; Millward et al., 1993b; Balthazor et al., 1997).

The enhanced electric field produces a family of gravity-wave disturbances in the thermosphere that propagate preferentially meridionally (both poleward and equatorward) from each auroral zone disturbance. 
For clarity and brevity, this study of the effects of AGWs and TIDs at equatorial latitudes will henceforth ignore the initially poleward-travelling waves, and refer to the equatorially-travelling waves as "northward-travelling' (originating in the Southern Hemisphere) and 'southward-travelling' (originating in the Northern Hemisphere) respectively. The waves interfere at the magnetic equator and pass through to the opposite hemispheres with unchanged velocity; this is consistent with observations (Heisler, 1958).

Figure 1 shows the signature of the AGWs at a fixed pressure surface denoted as H14 (13 scale heights above an imposed flat lower boundary of $1 \mathrm{~Pa}$ at $80 \mathrm{~km}$ ), corresponding to an approximate altitude of $380 \mathrm{~km}$, just below the F2 peak. The wave structure in both waves, consisting of a leading peak and a trailing trough, is clearly defined and stable; the waves propagate with approximately equal speeds of about $700 \mathrm{~m} \mathrm{~s}^{-1}$. At the magnetic equator, the pressure waves interfere and then pass into the opposite hemisphere.

Figure 2 shows the thermospheric signature of the AGWs at a lower fixed pressure surface denoted as H10, at an approximate altitude of $190 \mathrm{~km}$. The same peaktrough structure as at pressure level $\mathrm{H} 14$ is seen; there is also evidence for a secondary wave originating in each hemisphere, interfering over the magnetic equator at around 16.30 UT, but these secondary waves have a much smaller amplitude.

Figure 3 shows the thermospheric effects at a fixed pressure level denoted as $\mathrm{H} 08$, at approximately $135 \mathrm{~km}$, in the $\mathrm{E}$ region. The 'fast' waves visible at pressure surface $\mathrm{H} 14$ are seen, at a much reduced amplitude, and 'slow' waves with larger relative magnitudes are seen, having velocities of approximately $500 \mathrm{~m} \mathrm{~s}^{-1}$ in each case. The interference pattern suggests also the presence of yet smaller-amplitude waves interfering constructively at the magnetic equator at around 16.30 UT, possibly

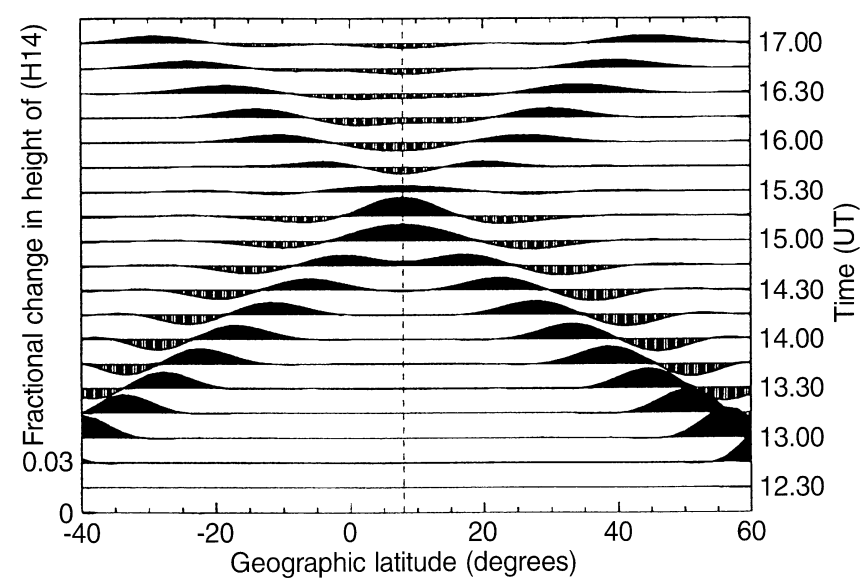

Fig. 1. Change in the height, relative to steady state conditions, of a fixed pressure level at approximately $380 \mathrm{~km}$ (just below the F2 peak). An increase in the height of the fixed pressure level is shown in heavy shading, whilst a decrease is shown by light shading. The AGW is shown at 20 times from 12.15 UT to 17.00 UT; each plot is offset up the $\mathrm{y}$-axis for clarity. The vertical dashed line denotes the magnetic equator

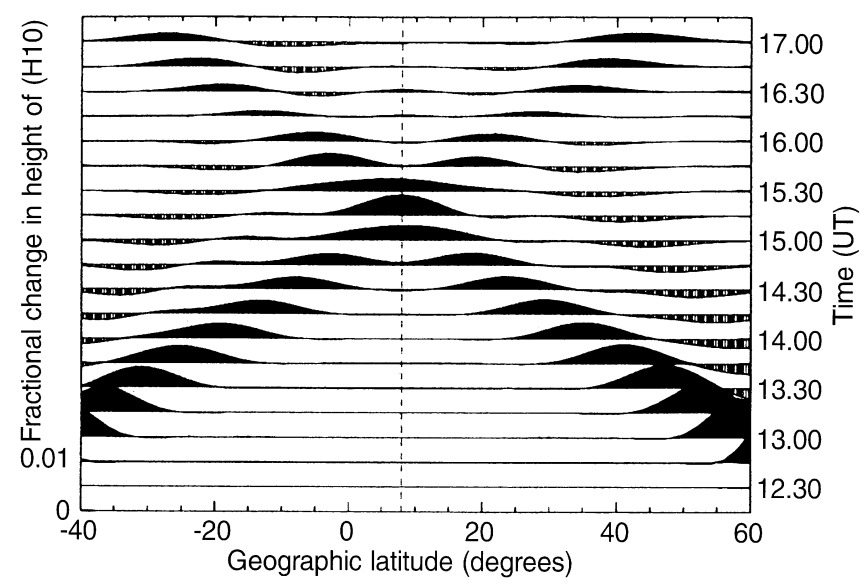

Fig. 2. As Fig. 1, except at a fixed pressure level at approximately $200 \mathrm{~km}$. Two waves of different velocities can be seen superimposed, from both the northern disturbance and the southern disturbance; the 'fast' wave seen in Fig. 1 and a slower 'intermediate' wave

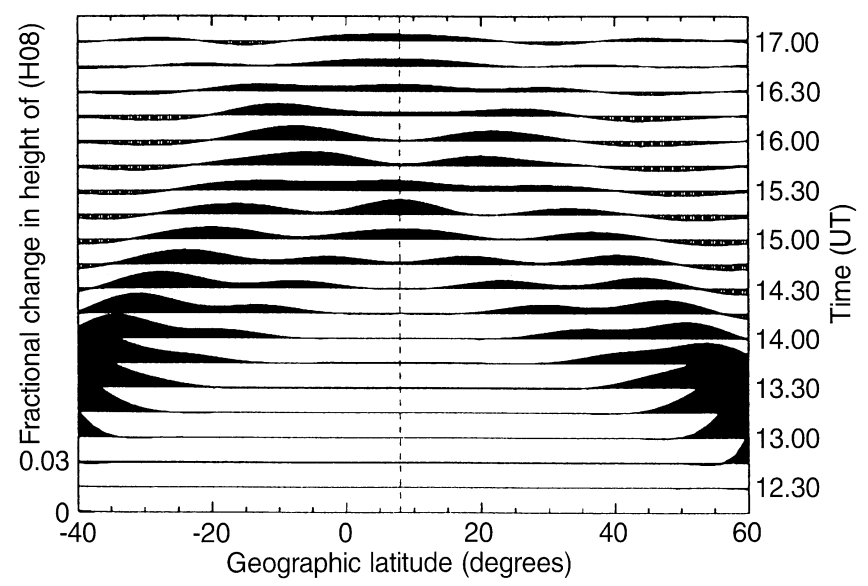

Fig. 3. As Fig. 1, except at a fixed pressure level at approximately $135 \mathrm{~km}$ (in the $\mathrm{E}$ region). Three waves of different velocities from both the northern disturbance and the southern disturbance can be observed: the fast, intermediate and slow waves described in the text

corresponding to the intermediate waves visible in Fig. 2.

At the equator, the AGWs interfere and continue through into the opposite hemisphere, with a clear symmetry about the magnetic equator. Only the slower $\mathrm{AGW}$ has a peak-trough structure, although it is supposed that the trough of the faster wave is interfering with the peak of the slower wave. This complex interference pattern means that the effects of the thermosphere on the plasmasphere at lower altitudes are difficult to interpret.

Considering the fast waves at fixed pressure surface $\mathrm{H} 14$, the wave period $\tau$ increases from approximately $55 \mathrm{~min}$ at $13.15 \mathrm{UT}$ to approximately $75 \mathrm{~min}$ at 14.30 UT (consistent with the characteristic period of thermospheric AGWs: Hines, 1960; Richmond, 1978; Lewis et al., 1996). The corresponding horizontal wavelength $\lambda_{x}$ is approximately $2300 \mathrm{~km}$, increasing to $3100 \mathrm{~km}$. The wave amplitude decays exponentially with an attenuation distance (where the wave amplitude decays by a 
factor e) of about $1500 \mathrm{~km}$. This fast wave therefore corresponds to an imperfectly ducted, or 'leaky', fundamental internal gravity wave mode (identified analytically by, among others, Thome, 1968 and Francis, 1973), ducted horizontally by the temperature gradient in the thermosphere, with energy propagating vertically up and dissipating at the top of the atmosphere. The intermediate and slow waves in Figs. 2 and 3 have been identified with similar imperfectly ducted modes, present due to the temperature gradient of the model that is effectively multiply-stepped compared with the two-layer model of Francis (1973).

\subsection{The resultant TIDs}

The TID is parameterised here by examining several quantities: hmF2 and $\mathrm{NmF} 2$, the height and electron density respectively of the $\mathrm{F} 2$ peak down the line of longitude $162^{\circ} \mathrm{E}$; the vertical profile of $\mathrm{N}_{e}$ both on the magnetic equator and at locations 10 degrees north and south; and the ion velocities along the plasma flux tubes, a more basic parameter in the structure and formation of the TID. It is noted that the behaviour of the 'observed' TID depends significantly on the parameter chosen to characterise it.

As the plasma is constrained to move along the magnetic field lines which in general have a vertical component (except at the magnetic equator), it is clear that the mainly horizontal motions of the thermosphere due to the AGW (the pressure-driven horizontal wind) will tend to force the ions both vertically and horizontally. An AGW propagating equatorward through midlatitudes will tend to drive ions up the field lines before it and down the field lines behind it; an AGW propagating poleward through mid-latitudes will reverse these effects. Figure 4 shows $\delta$ hmF2, the fractional change in hmF2 from diurnally steady-state conditions. Prior to the TIDs reaching the equator, the fractional change in the height of the F2 peak is larger than the corresponding fractional change in the thermospheric fixed pressure

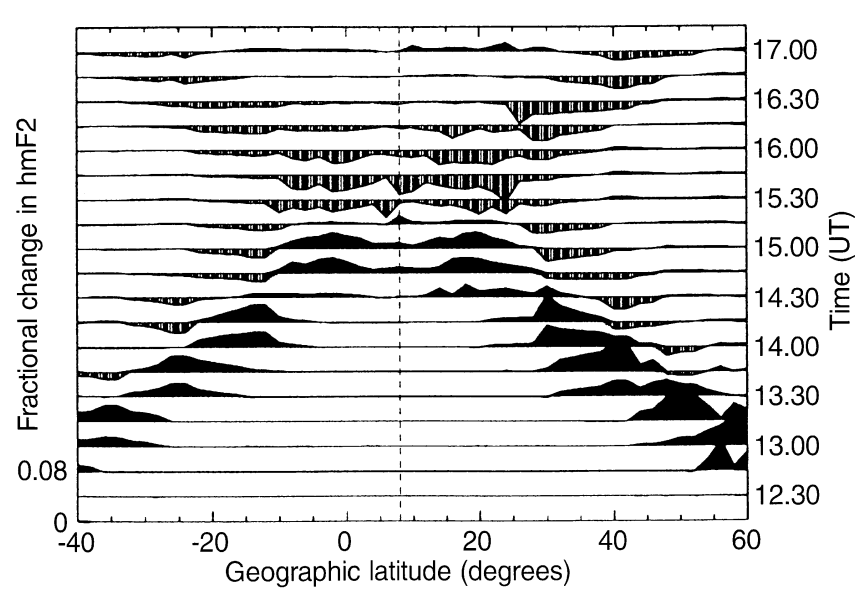

Fig. 4. Change in hmF2 (the height of the F2 peak), relative to steadystate conditions. The format of the plots is identical to that of Fig. 1 level at a similar height, by a factor of between two and three. There is still a distinct leading peak and following trough structure to the TID, corresponding to the signature of the AGW in the thermosphere, but the wave is less clearly defined than the AGW and the amplitude and duration of the trough is much smaller than that of the peak. After the AGW has passed through the equator, the leading peak of the associated TID becomes a trough of longer duration but smaller amplitude. It is noted that $\delta \mathrm{hmF} 2$ at the magnetic equator is small but not zero. Although ions cannot move perpendicularly to the field lines (apart from imposed $\mathrm{E} \times \mathrm{B}$ drift), they may be driven up the field lines from lower altitudes, altering the shape and distribution of the F2 peak, which has the effect of changing hmF2.

The global model used has the electron density set equal to the total ion density, which below about $420 \mathrm{~km}$ includes the molecular species $\mathrm{N}^{+}, \mathrm{O}_{2}^{+}, \mathrm{NO}^{+}$and $\mathrm{N}_{2}^{+}$ and above that height is simply $\mathrm{H}^{+}$and $\mathrm{O}^{+}$. The fractional change in the electron density at the F2 peak due to the AGW, $\delta \mathrm{NmF} 2$, is shown in Fig. 5. Travelling through mid-latitudes towards the equator, there is little change in $\mathrm{NmF}$, which tends to be in antiphase with the changes in hmF2, as predicted by Fesen et al. (1989). At $14.30 \mathrm{UT}$, the driving AGWs are forcing ions up the field lines from lower altitudes, increasing NmF2; after 15.30 UT, the ions are being driven back down the field lines by the fast AGWs: there is an increase in NmF2 around $15^{\circ}$ north and south of the magnetic equator (here, $25^{\circ} \mathrm{N},-10^{\circ} \mathrm{S}$ geographic) where the downwarddriven ions meet the upward-driven ions of the slower TIDs. At equatorial latitudes, however, NmF2 remains depleted, due to ions trapped below $300 \mathrm{~km}$ by the complex action of neutral winds generated by the slower gravity waves. It is noted that this change in NmF2 is due only to motion of the ions along the field lines; the change in the production/loss rate due to the change in temperature is very small. This behaviour may be compared to that of Fig. 6, where the source disturbance was only in the northern auroral electrojet. Plasma is pushed over the field lines into the Southern Hemisphere

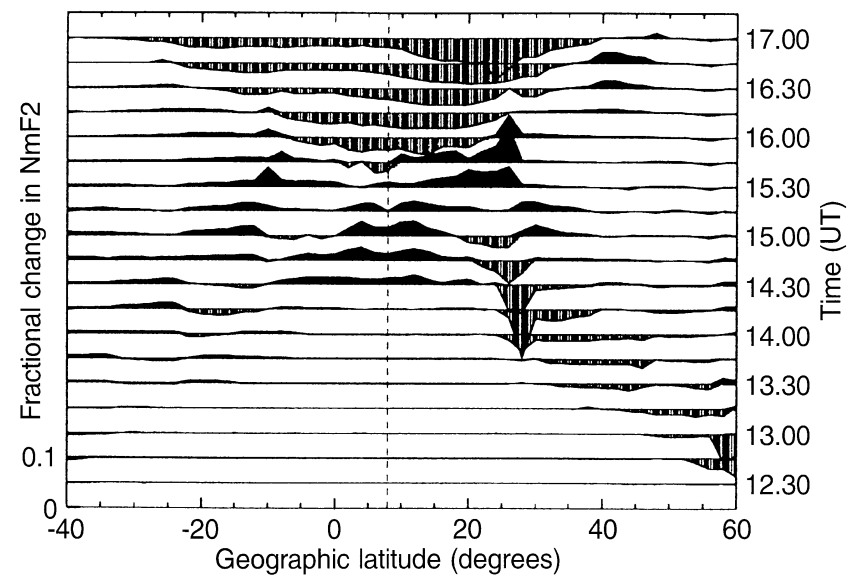

Fig. 5. Change in $\mathrm{NmF} 2$ (the density of the $\mathrm{F} 2$ peak), relative to steady-state conditions. The format of the plots is identical to that of Fig. 1 


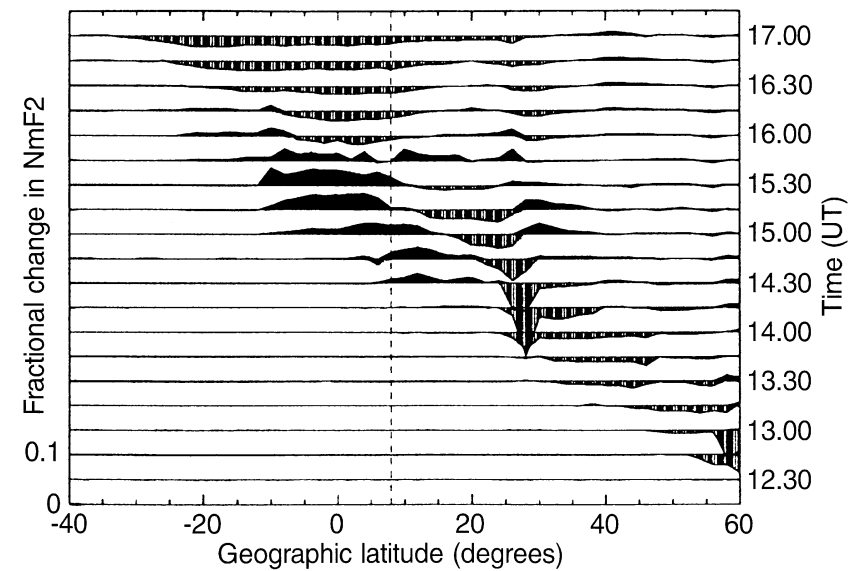

Fig. 6. Change in $\mathrm{NmF} 2$ (the density of the F2 peak), relative to steady-state conditions, with only a northern auroral disturbance. The format of the plots is identical to that of Fig. 1

by the fast AGW, and in the Southern Hemisphere remains at lower altitudes as the slower waves pass through.

The signature of the TID clearly depends on the parameterisation used. Examining hmF2 (Fig. 4) shows a pattern that is both relatively symmetric latitudinally about the equator, and relatively stable in the spatial correspondence with the forcing AGW. In contrast, the change in NmF2 (Fig. 5) is distinctly anti-symmetric latitudinally about the equator, and has a very poor spatial correspondence to the forcing AGWs; this is also seen examining only a Northern-Hemisphere disturbance (Fig. 6), which shows the poor spatial correlation between the AGW and the TID.

The vertical profile of $\mathrm{N}_{e}$ at the magnetic equator is shown in Fig. 7. The lowering of the atmosphere during the night is clearly shown. The fractional change in $\mathrm{N}_{e}$ is shown in Fig. 8. The signature of the TID is approximately of equal magnitude both below and above the

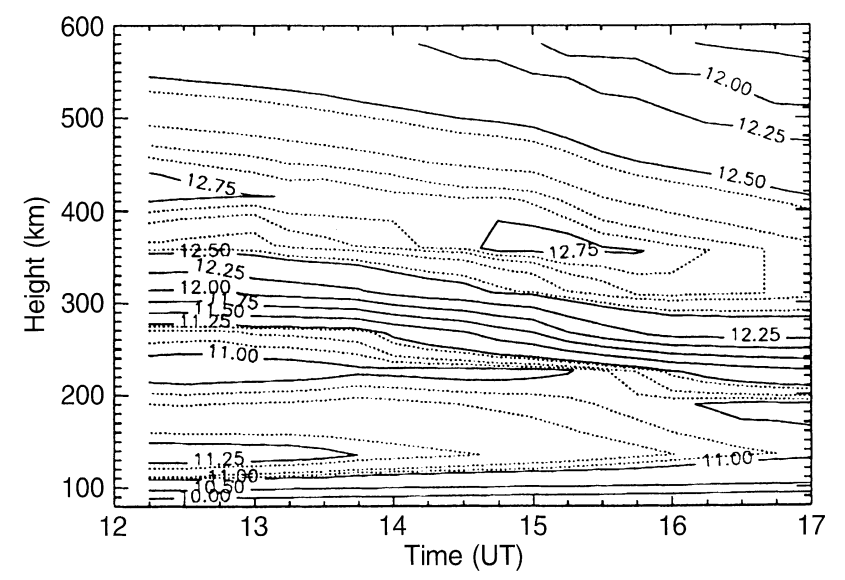

Fig. 7. Vertical profile of electron concentration at the magnetic equator at $162^{\circ} \mathrm{E}$, from $12.15 \mathrm{UT}$ to $17.00 \mathrm{UT}$. Values are $\log _{10} \mathrm{~N}_{e}$. Equally spaced contours are shown as solid lines, whilst dotted lines are intermediate arbitrary values chosen to show structure. The contours at 10.25 and 10.75 have been omitted for clarity. The 'fast' AGW passes over the magnetic equator between 14.45 and $16.00 \mathrm{UT}$

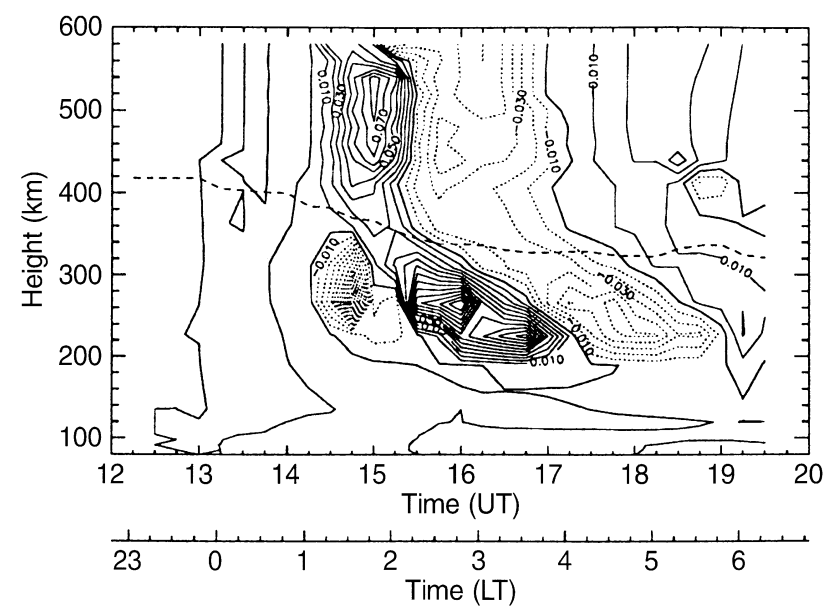

Fig. 8. Vertical profile of fractional change in electron concentration at the magnetic equator at $162^{\circ} \mathrm{E}$, from 12.15 UT to $19.50 \mathrm{UT}$. Contours are spaced every $\delta \mathrm{N}_{e}=0.01$. The solid (dotted) lines are positive (negative) contours. The broken line running left to right across the plot shows the height of the F2 peak, hmF2

F2 peak. This may be contrasted with Fig. 9 showing predictions for the wave passing through a low-latitude station $\left(24^{\circ} \mathrm{N}\right.$ geographic), where the 'slow' southwardpropagating wave interferes with the 'fast' northward propagating wave (in a similar way to the two 'fast' waves interfering at the equator). The waves interfere at 15.45 UT (02.33 LT). At this low latitude, the signature of the TID is largest below the F2 peak, with the signature in the upper F-region proportionally smaller.

Figure 10 shows the equatorial data as a series of individual vertical profiles. At the equator, the vertical profile initially exhibits a sharply peaked decrease in $\mathrm{N}_{e}$ centred around $260 \mathrm{~km}$ (considerably below the F2 peak) with an increase in $\mathrm{N}_{e}$ above $400 \mathrm{~km}$. This situation then reverses between 15.15 and 15.30 UT (02.17 LT), when $\mathrm{N}_{e}$ increases rapidly below $350 \mathrm{~km}$ and

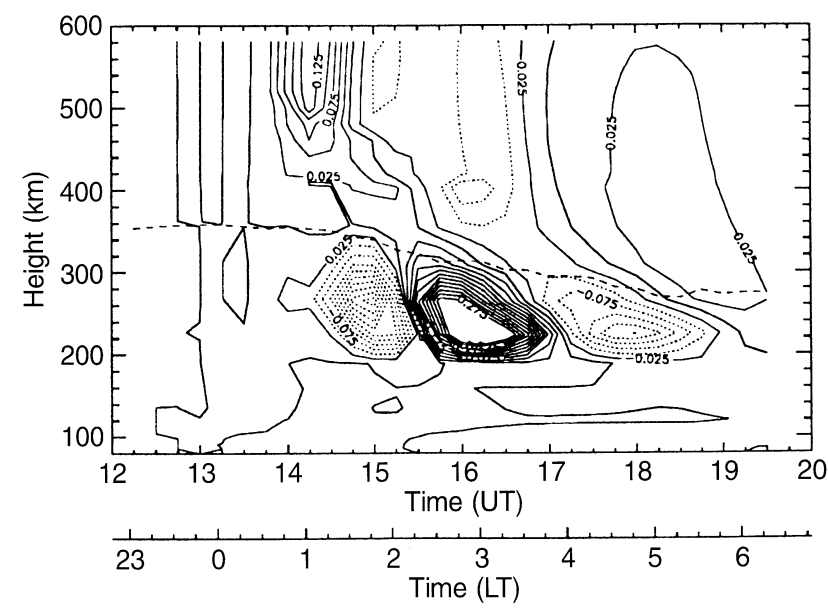

Fig. 9. Low latitude vertical profile of fractional change in electron concentration at $24^{\circ} \mathrm{N}$ (geographic), ( $\left(16^{\circ} \mathrm{N}\right.$ magnetic), $162^{\circ} \mathrm{E}$ (geographic), from 12.15 UT to 19.50 UT. Contours are spaced every $\delta \mathrm{N}_{e}=0.025$. The denotation of the contours and hmF2 are described in Fig. 8 


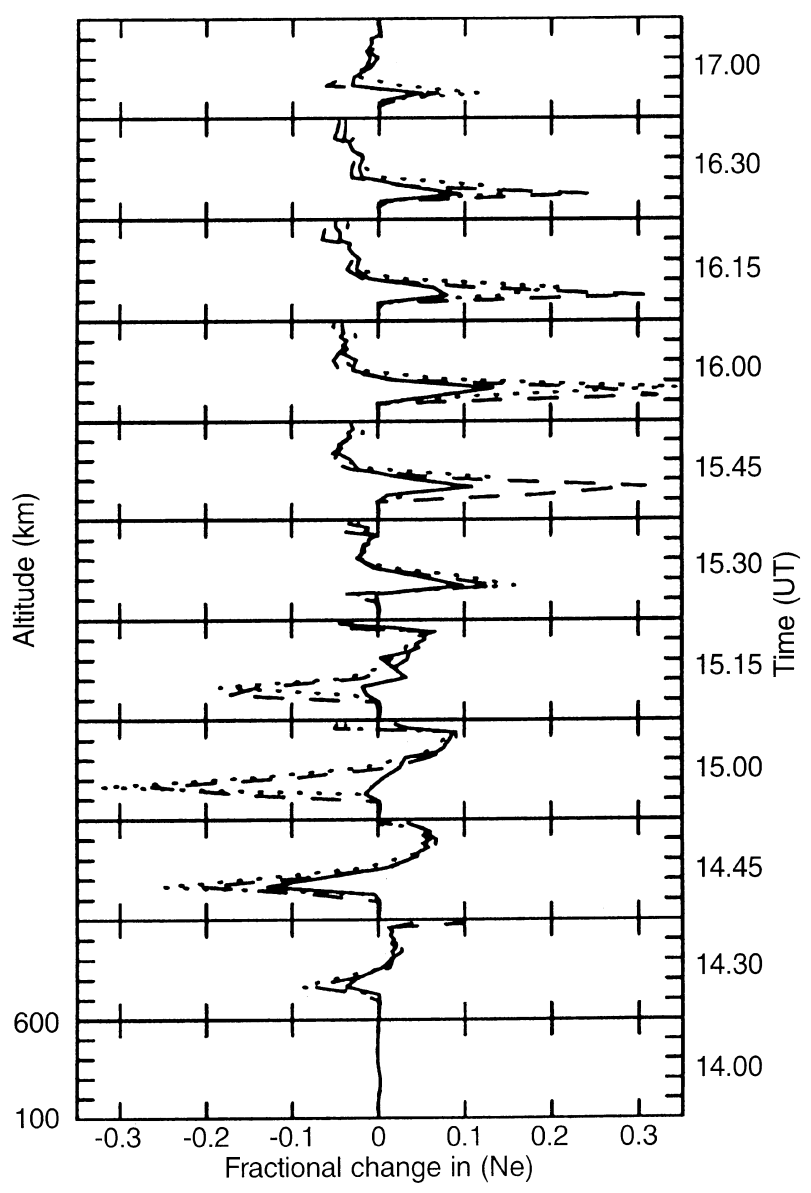

Fig. 10. Change in $\mathrm{N}_{e}$ at the magnetic equator from 100 to $600 \mathrm{~km}$ as the AGWs pass overhead, on the line of geographic longitude $162^{\circ}$ east. The solid lines show the fractional change in $\mathrm{N}_{e}$ from diurnally steady-state conditions at successive times from 14.00 UT to 17.00 UT. Each interval is $15 \mathrm{~min}$, apart from the first and last intervals which are $30 \mathrm{~min}$. The dashed line in each plot shows fractional change in $\mathrm{N}_{e}$ at a point $10^{\circ}$ north of the magnetic equator, whilst the dotted line shows the fractional change in $\mathrm{N}_{e} 10^{\circ}$ south of the magnetic equator

decreases above that altitude. At ten degrees north and south of the magnetic equator, the vertical profile is similar, but the fractional changes in $\mathrm{N}_{e}$ at around $260 \mathrm{~km}$ are considerably larger than at the equator; this is a consequence of the larger dip angle of the magnetic field at mid-latitudes, where the mainly horizontal forcing neutral winds may drive the ions with a larger vertical component of motion. The explanation of the reversal of the sign of the fractional change in $\mathrm{N}_{e}$ may be found by examining the vertical wavelength of the forcing AGW. From the standard analysis of atmospheric wave propagation (Hines, 1960), rearranging terms and taking the approximation $\omega \gg \omega_{a}$ (the acoustic wave frequency), the dispersion relation may be written as

$\lambda_{z}^{2}=\left(\frac{\tau_{g}^{2}}{\tau^{2}-\tau_{g}^{2}}\right) \lambda_{x}^{2}$

where $\lambda_{x}$ is the horizontal wavelength, $\lambda_{z}$ is the vertical wavelength, $\tau$ is the wave period and $\tau_{g}$ is the Brunt-
Väisälä period. Kelley (1989) gives $\tau_{q}$ at $380 \mathrm{~km}$ as lying between 12 and $17 \mathrm{~min}$; taking an approximation of $15 \mathrm{~min}$, we obtain $\lambda_{z} \approx 650 \mathrm{~km}$ at this altitude. This is large compared with the thickness of the F2 peak, and so the passage of the AGW lifts the whole of the F2 peak in phase; the change in sign of the fractional change in $\mathrm{N}_{e}$ thus simply corresponds to the change in sign of the gradient of the F2 peak (after Thome, 1968). Figure 11 shows the same vertical profile at the equator over an extended time until 18.45 UT. The disturbance is still present $5 \mathrm{~h}$ after the 'fast' waves interfere at the equator and decays slowly; the ions that were previously trapped at lower altitudes are now slowly moved back to the upper F-region. Evidence can also be seen in Fig. 11 of vertical wavelengths shorter than $650 \mathrm{~km}$; it is suggested that these are harmonics of the wavelengths associated with both the 'fast' AGW and with the subsequent 'slower' AGWs interfering.

The effect of the AGWs on the fixed pressure surfaces, the neutral winds and the ion winds are shown in Figs. 12, 13 and 14, giving cross-sections of the atmospheric wind patterns at 14.30 UT, 15.15 UT and 16.15 UT respectively. As the two AGWs approach the equator at $14.30 \mathrm{UT}(01.18 \mathrm{LT})$, the neutral atmosphere is lifted, pushing the ions up along the field lines as in Fig. 12. Here, the AGWs have a strong equatorward wind caused by the pressure wave, driving the ions up

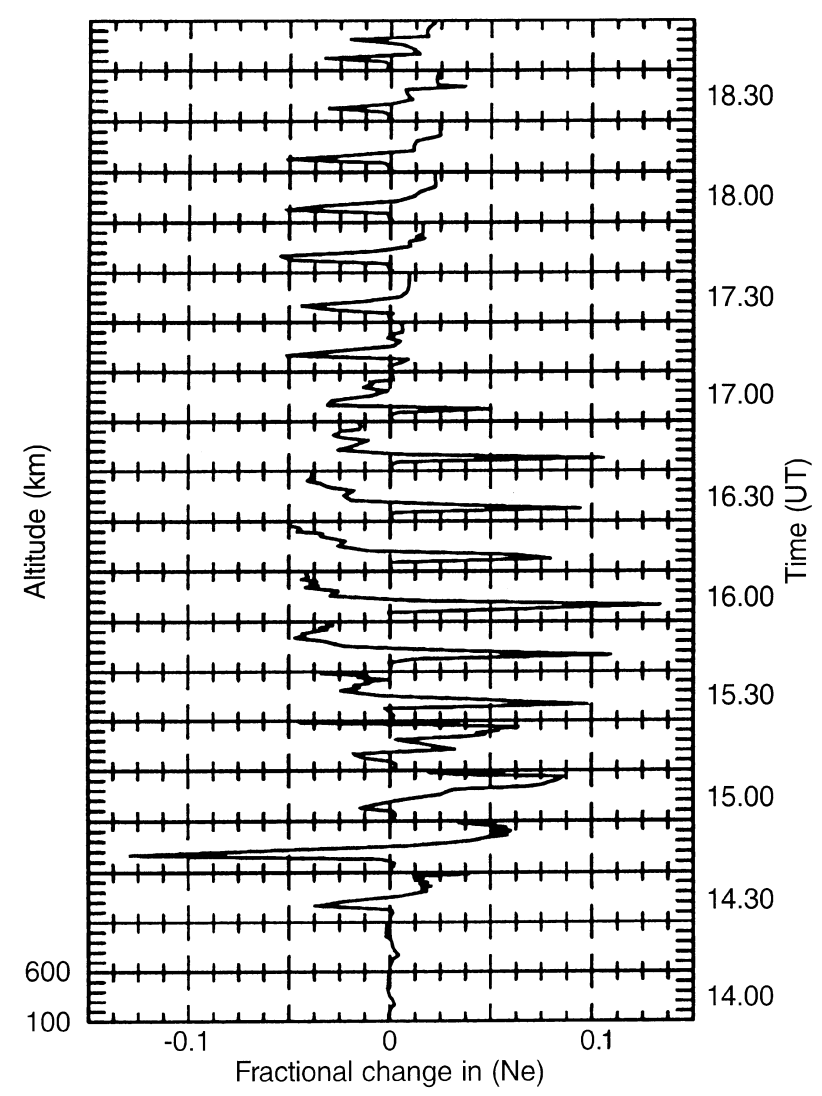

Fig. 11. Change in $\mathrm{N}_{e}$ at the magnetic equator from 100 to $600 \mathrm{~km}$ as the AGWs pass overhead, on the line of geographic longitude $162^{\circ}$ east. The vertical profiles show the fractional change in $\mathrm{N}_{e}$ at the magnetic equator every $15 \mathrm{~min}$ from $14.00 \mathrm{UT}$ to $18.45 \mathrm{UT}$ 

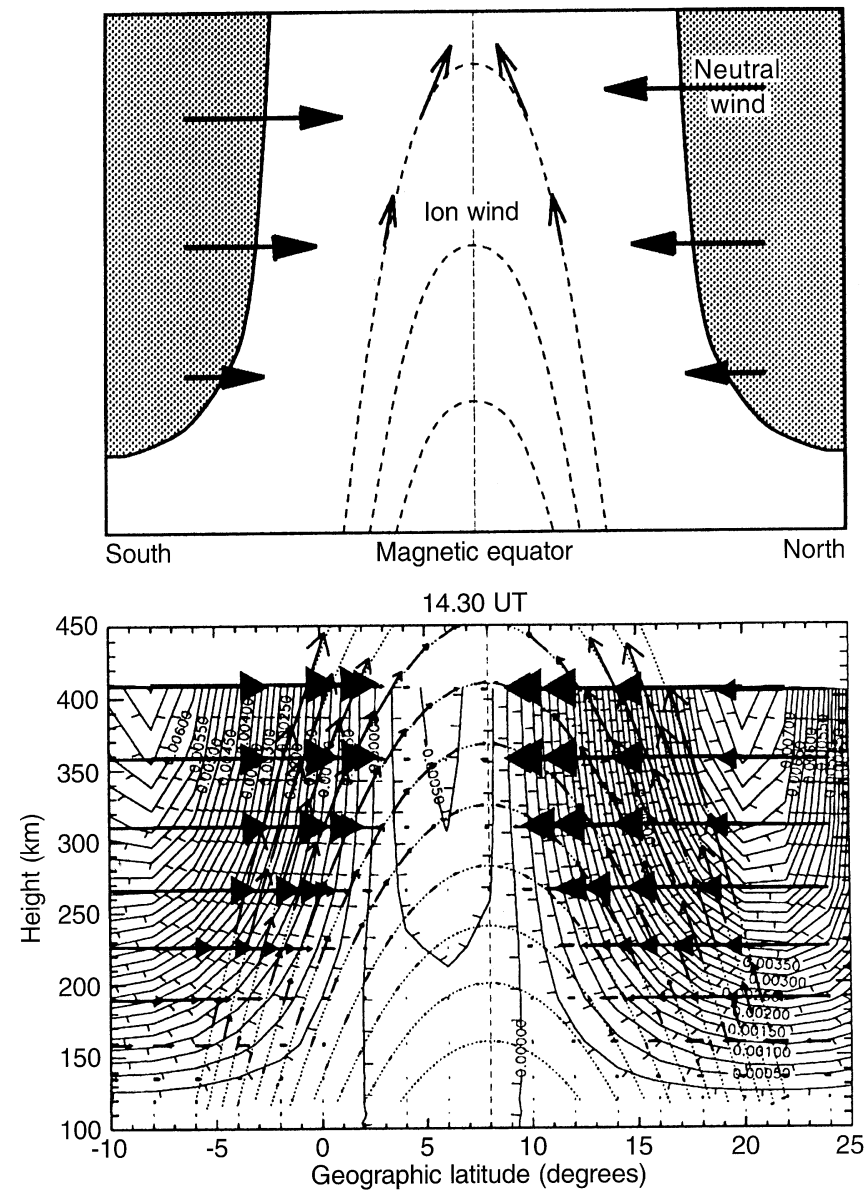

Fig. 12. A slice through the atmosphere at a fixed longitude on the geographic meridian $162^{\circ} \mathrm{E}$, from $10^{\circ} \mathrm{S}$ to $25^{\circ} \mathrm{N}$ and from $100 \mathrm{~km}$ to $450 \mathrm{~km}$ in altitude, at UT 14.30. The upper plot shows a schematic of the main behaviour of the plot, and is not to scale. Shaded areas show regions where the height of fixed pressure levels is increased due to the passage of the AGW, whilst solid (hollow) arrows show changes in the neutral (ion) winds. The contour plot below (with arbitrary levels of contours chosen to illustrate the structure) shows the fractional change (from diurnal steady-state conditions) of the height of the thermospheric fixed pressure levels, due to the passage of the AGWs. The thick solid arrows show the direction and comparative magnitude of the change in the component of the neutral wind in this meridian. The looped dotted lines show the projections of the nearest calculated adjacent flux tubes onto the geographic meridian. The thin open arrows show the direction and magnitude of the change in the ion wind due to this fluctuation from steady state conditions. Although the size of each arrow is proportional to the magnitude of the wind component, the magnitude of the ion wind arrows has been multiplied by a factor of 10 to show more clearly the direction of the (smaller) ion wind

the field lines. The horizontal wind increases with altitude (corresponding to the decrease in pressure). The sloping phase front of the AGW is evident below $250 \mathrm{~km}$, indicating an upward flow of energy.

At 15.15 UT $(02.03 \mathrm{LT})$, the two AGWs have combined over the magnetic equator. Above $250 \mathrm{~km}$, the pressure gradient is now driving a horizontal neutral wind polewards into each hemisphere, forcing the ions back down the flux tubes (and enhancing the equatorial anomaly in each hemisphere). Below $250 \mathrm{~km}$ there is interference between the slower and faster AGWs,
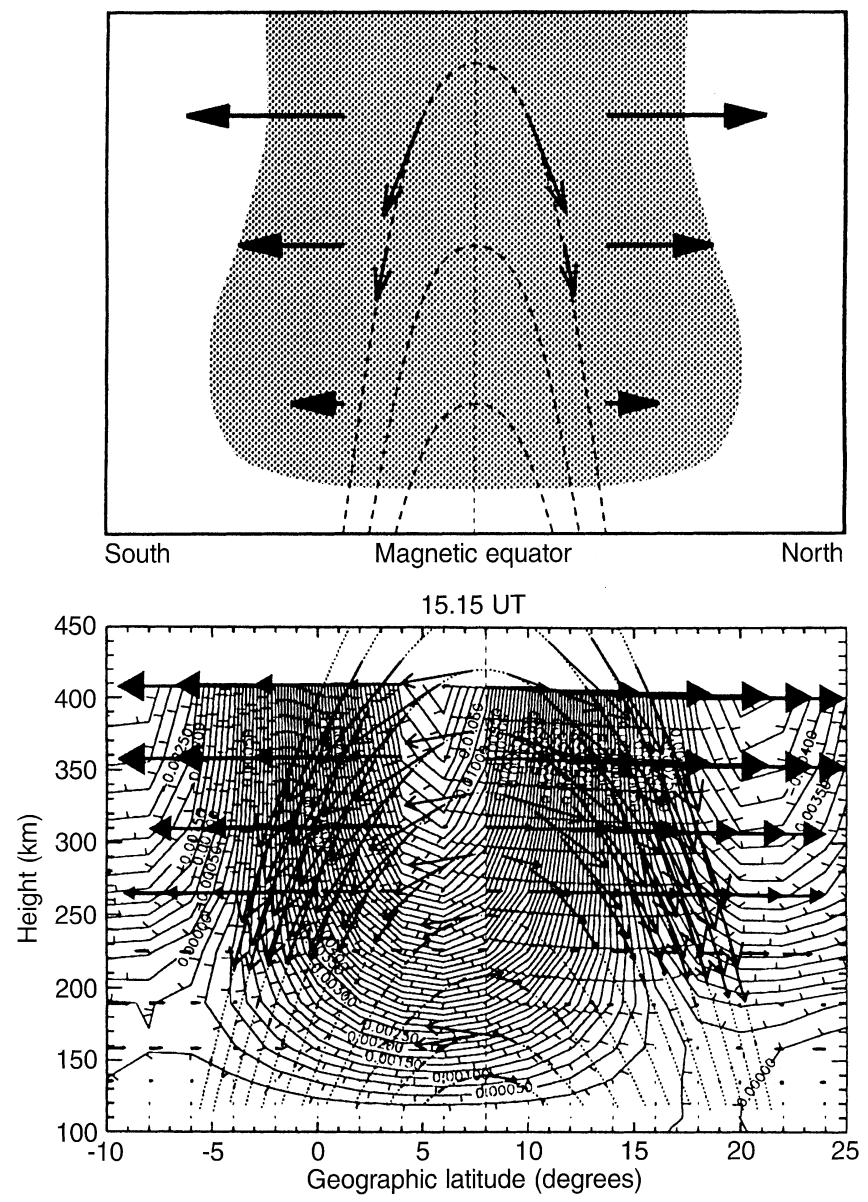

Fig. 13. As Fig. 12, except the slice is taken at 15.15 UT. The upper panel shows the main features of the plot. The contour levels (lower panel) are identical to Fig. 12

resulting in smaller pressure gradients. The neutral winds are reduced and there are residual ion motions from the previous timestep.

At 16.15 UT (03.03 LT), the two fast AGWs have now passed into the opposing hemispheres. Above $250 \mathrm{~km}$, each wave is now distinct, the pressure gradients forcing the neutral atmosphere meridionally away from each wave, and the trailing edges of the AGWs are seen. Ions in the upper sections of the flux tubes are consequently driven upwards once again (although it is noted that there is a net neutral wind southward across the magnetic equator, forcing ions over the top of the flux tubes). In the region above about $180 \mathrm{~km}$ but below $250 \mathrm{~km}$, the slow AGWs are interfering and resulting in a pressure gradient away from the magnetic equator, forcing a poleward neutral wind into each hemisphere.

\section{Concluding remarks}

The global fully coupled thermosphere-ionosphereplasmasphere model has been used to simulate AGWs originating from an electric-field enhancement at auroral latitudes, and propagating to equatorial latitudes, along with the associated TID signatures. The AGWs 


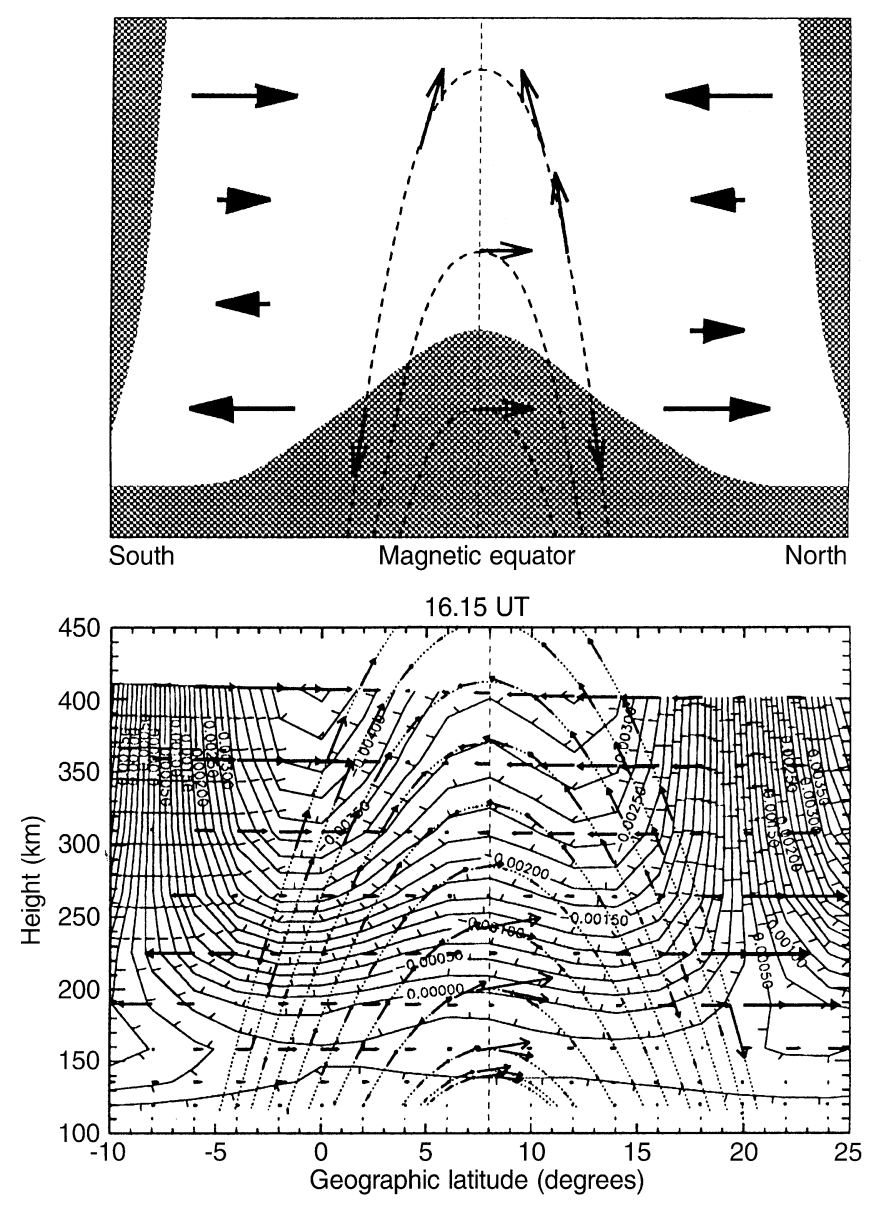

Fig. 14. As Fig. 12, except the slice is taken at 16.15 UT. The upper panel shows the main features of the plot. The contour levels (lower panel) are identical to Fig. 12

are identified as a family of imperfectly ducted wavemodes. The fundamental mode of this family have been predicted by simple thermospheric models e.g. Francis, (1973) and the more complex coupled model used here predicts additional slower modes of smaller amplitude. These are in agreement with observations by Thome (1968) and Hajkowicz and Hunsucker (1987).

The behaviour of the AGWs and TIDs at the equator has been studied in detail and the complex observable characteristics of the TIDs are explained by the behaviour of the forcing horizontal neutral winds. Observing the ionosphere only at one height (e.g. NmF2 or hmF2) presents an incomplete picture of the family of TIDs. The conjugate AGWs originating in both north and south auroral zones interfere constructively at the equator and the magnitude of the TID is slightly increased, confirming the suggestion of Hajkowicz and Hunsucker (1987) and the statistical survey of Hajkowicz (1991). The TIDs interfere to produce a stationary and decaying perturbation both above and below the F2 peak; the increase in magnitude of the perturbation in the upper F-region at the equator compared to that at mid-latitudes is of greater magnitude than the similar increase in magnitude of the perturbation in the lower F-region. The perturbation above the F2 peak decays over a period of about $1 \mathrm{~h}$, whilst the perturbation below the F2 peak decays more slowly over a period of several hours. It is suggested that the persistence of the lower F-region disturbance is due to the arrival at the equator of pairs of slower AGWs generated by the original auroral source, which act to counter the decaying perturbation caused by the first 'fast' pair of AGWs. We also note this study has simulated a single impulsive event which generated a family of single large-scale gravity waves. Studies by e.g. Williams et al. (1988) have shown that there can be a periodicity to the source mechanism to produce an AGW wave-train, and such a wave-train could further prolong the decay of the lower F-region equatorial disturbance as suggested by Gross et al. (1984).

Acknowledgements. This work has been supported by the UK Science and Engineering Research Council and Particle Physics and Astronomy Research Council.

Topical Editor D. Alcaydé thanks H. Hocke and R. V. Lewis for their help in evaluating this paper.

\section{References}

Balthazor, R. L., R. J. Moffett, and G. H. Millward, a study of the Joule and Lorentz inputs in the production of atmospheric gravity waves in the upper thermosphere, (in press) Annales Geophysicae, 1997.

Bowman, G. G., Short-term delays in ionogram-recorded equatorial spread-F occurrence after solar and geomagnetic activity, Annales Geophysicae, 13, 1331, 1995.

Fesen, C. G., G. Crowley, and R. G. Roble, Ionospheric effects at low latitudes during the March 22, 1979, geomagnetic storm, J. Geophys. Res., 94, 5405, 1989.

Foster. J. C., An empirical electric field model derived from Chatanika radar data, J. Geophys. Res., 88, 981, 1983.

Francis, S. H., Acoustic-gravity modes and large-scale travelling ionospheric disturbances of a realistic, dissipative atmosphere, J. Geophys. Res., 78, 2278, 1973.

Fukao, S., Y. Yamamoto, W. L. Oliver, T. Takami, M. D. Yamanaka, M. Yamamoto, T. Nakamura, and T. Tsuda, Middle and upper atmosphere radar observations of ionospheric horizontal gradients produced by gravity waves, J. Geophys. Res., 98, 9443, 1993.

Fuller-Rowell, T. J., A two-dimensional, high-resolution, nestedgrid model of the thermosphere. 1. Neutral response to an electric field "spike", J. Geophys. Res., 89, 2971, 1984.

Fuller-Rowell, T. J., and D. S. Evans, Height-integrated Pederson and Hall conductivity patterns inferred from the TIROSNOAA satellite data, J. Geophys. Res., 92, 7606, 1987.

Fuller-Rowell, T. J., D. Rees, S. Quegan, R. J. Moffett, M. V. Codrescu, and G. H. Millward, A coupled thermosphereionosphere model (CTIM); STEP Handbook on Ionospheric Models, Ed. RW Schunk, Utah State University, 217, 1996.

Gross, S. H., C. A. Reber, and F. T. Huang, Large-scale waves in the thermosphere observed by the AE-C satellite, IEEE Trans. on Geoscience Remote Sensing, GE-22, 340, 1984.

Hajkowicz, L. A., A global study of large scale travelling ionospheric disturbances (TIDs) following a step-like onset of auroral substorms in both hemispheres, Planet. Space Sci., 38, 913, 1990.

Hajkowicz, L. A., Auroral electrojet effect on the global occurrence pattern of large-scale travelling ionospheric disturbances, Planet. Space Sci., 39, 1189, 1991.

Hajkowicz, L. A., Ionospheric response to auroral substorms during sunspot maximum (1980-82), Annales Geophysicae, 13, 95, 1995. 
Hajkowicz, L. A., and R. D. Hunsucker, A simultaneous observation of large-scale periodic TIDs in both hemispheres following an onset of auroral disturbances, Planet. Space Sci., 35, 785, 1987.

Heisler, L. H., Anomalies in ionosonde records due to travelling ionospheric disturbances, Austr. J. Phys. 11, 79, 1958.

Hines, C. O., Internal atmospheric gravity waves at ionospheric heights, Can. J Phys., 38, 1441, 1960.

Hines, C. O., The upper atmosphere in motion, Geophysical Monograph, American Geophysical Union, 1974.

Hocke, K., and K. Schlegel, A review of atmospheric gravity waves and travelling ionospheric disturbances: 1982-1995, Annales Geophysicae, 14, 917, 1996.

Hunsucker, R. D., Atmospheric gravity waves generated in the highlatitude ionosphere: a review, Rev. Geophys. Space Phys., 20, 293, 1982.

Igarashi, K., A. Ohtani, T. Maruyama, M. Tanaka, M. Kamata, I. Nishimuta, and N. Kobayashi, Ionospheric disturbance features in ionograms associated with low-latitude aurorae, Proc NIPR Symposium Upper Atmosphere Physics, 4, 70, 1991.

Jing, N., and R. D. Hunsucker, A theoretical investigation of sources of large and medium scale atmospheric gravity waves in the auroral oval, J. Atmos. Terr. Phys., 55, 1667, 1993.

Johnson, F. S., W. B. Hanson, R. R. Hodges, W. R. Coley, G. R. Carignan, and N. W. Spencer, Gravity waves near $300 \mathrm{~km}$ over the polar cap, J. Geophys. Res., 100, 23993, 1995.

Kelley, M. C., Earth's Ionosphere, Academic Press, 1989.

Kirchengast, G., K. Hocke, and K. Schlegel, The gravity wave-TID relationship: insight via theoretical model-EISCAT data comparison, J. Atmos. Terr. Phys., 58, 233, 1996.

Lewis, R. V., P. J. S. Williams, G. H. Millward, and S. Quegan, The generation and propagation of atmospheric gravity waves from activity in the auroral electrojet, J. Atmos. Terr. Phys., 58, 807, 1996.

Millward, G. H., R. J. Moffett, S. Quegan, and T. J. Fuller-Rowell, Effects of an atmospheric gravity wave on the midlatitude ionospheric F Layer, J. Geophys. Res., 98, 19173, 1993a.

Millward, G. H., S. Quegan, R. J. Moffett, T. J. Fuller-Rowell, and D. Rees, A modelling study of the coupled ionospheric and thermospheric response to an enhanced high-latitude electric field event, Planet. Space Sci., 41, 45, 1993 b.

Millward, G. H., R. J. Moffett, S. Quegan, and T. J. Fuller-Rowell, A coupled thermosphere-ionosphere-plasmasphere model CTIP, In STEP Handbook on Ionospheric Models, Ed. RW Schunk, Utah State University, 239, 1996.

Pryse, S. E., C. N. Mitchell, J. A. T. Heaton, and L. Kersley, Travelling ionospheric disturbances imaged by tomographic techniques, Annales Geophysicae, 13, 1325, 1995.

Richmond, A. D., Gravity wave generation, propagation, and dissipation in the thermosphere, J. Geophys. Res., 83, 4131, 1978.

Sterling, D. L., W. H. Hooke, and R. Cohen, Travelling ionospheric disturbances observed at the magnetic equator, J. Geophys. Res., 76, 3777, 1971.

Thome, G., Long-period waves generated in the polar ionosphere during the onset of magnetic storms, J. Geophys. Res., 73, 6319, 1968.

Williams, P. J. S., G. Crowley, K. Schlegel, T. S. Virdi, I. McCrea, G. Watkins, N. Wade, J. K. Hargreaves, T. Lachlan-Cope, H. Muller, J. E. Baldwin, P. Warner, A. P. van Eyken, M. A. Hapgood, and A. S. Rodger, The generation and propagation of atmospheric gravity waves observed during the worldwide atmospheric gravity-wave study (WAGS). J. Atmos. Terr. Phys., 50, 323, 1988. 